\title{
Impurity Profiling of Solid Oral Drug Products to Sail through GDUFA-II Requirements
}

\author{
Raghuram Pannala \\ Quality Assurance and Regulatory Affairs, ScieGen Pharmaceuticals, Inc., Hauppauge, NY, USA \\ Email: ramp.raghu@gmail.com
}

How to cite this paper: Pannala, R. (2018) Impurity Profiling of Solid Oral Drug Products to Sail through GDUFA-II Requirements. American Journal of Analytical Chemistry, 9, 187-209.

https://doi.org/10.4236/ajac.2018.94016

Received: February 8, 2018

Accepted: April 6, 2018

Published: April 9, 2018

Copyright $\odot 2018$ by author and Scientific Research Publishing Inc. This work is licensed under the Creative Commons Attribution International License (CC BY 4.0).

http://creativecommons.org/licenses/by/4.0/

\section{c) (i) Open Access}

\begin{abstract}
Defining impurity profile is key element to ensure safe, efficacious and quality human drugs. Impurity profiling changed/transformed drastically over the years. Guidelines, specifications and requirements are evolving. Initially impurity profiling was based on simple methods later by degradation studies, then to understand drug strength and efficacy chiral impurities and stereo isomers were included followed by residual solvents, polymorphic forms, genotoxic impurity studies. Currently, elemental impurities are the latest addition. As per the GDUFA II guidelines to improve review efficiency and reduce review cycles, data requirements have changed. Based on recent guidance and review points, Impurity profiling has significant importance in ANDA filing and to ensure approval within 10 months (first cycle approval) which is an exiling aspect for industries to enter into the generic market quickly. Hence, Impurity profile is a key aspect scientifically, regulatory wise and commercially also. This is a review article on impurity profiling of Solid oral drug substances and products as per GDUFA II requirements the reference documents for the review are ICH guidance, relevant FDA GDUFA guidance and common industry practices.
\end{abstract}

\section{Keywords}

Impurity Profile, Degradation, Stereo Isomers, Genotoxic, Polymorphic, Elemental Impurities, FDA, GDUFA II, Organic Impurities, Inorganic Impurities, RTR, Specifications

\section{Introduction}

Impurity is considered as any component of a drug substance that is not the 
chemical entity defined as the drug substance and in addition, for a drug product, any component that is not a formulation ingredient [1]. GDUFA II Guidance on good submission practices emphasizes the importance and requirements of impurity profiling in drug substances and drug products to improve review efficiency and reduce review cycles data requirements are revised [2]. Impurity profiling is the basis for determining, assuring quality, safety and efficacy of the drug substance and drug product. It has gained more significance in GDUFA environment and also in evolution of new guidance and review points [3]. Firms must ensure the highest quality and compliance of impurity profiling to safeguard first cycle approvals (10 months). The evolution and changes in impurities identification, quantification and control are a part of evolving quest of scientific community to deliver safe, effective and quality medicines.

Defining impurity profile of the drug substance (Active Pharmaceutical Ingredient) is the basis for impurity profiling of Drug product, however, it also considers excipients and formulation process. This review document will discuss the guidelines to be considered in categorizing the impurities, identifying the possible source, evaluation, analytical methods which can be used, qualifying impurity levels and proposing limits and common deficiencies anticipated in ANDA review [4]-[10].

Impurities in Drug substances and Drug product can be classified as follows as per the current guidance under GDUFA-II requirements

1) Organic Impurities

i) Process

ii) Degradation

iii) Chiral Impurities

2) Genotoxic Impurities (discussed as separate topic)

3) In Organic Impurities (Elemental impurities erstwhile Heavy metals)

4) Residual Solvents

5) Polymorphic Impurities

The evolution of impurities testing in drug substances and drug products over the period can be largely categorized as below (Figure 1).

A snap shot of the impurity profiling of drug substance and drug product is presented in the below illustration (Figure 2).

\section{Organic Impurities (Process and Drug Related)}

Organic impurities can arise during the manufacturing process, drug substance-excipient interactions, and/or storage of the drug substance, drug product [1]. They can be identified or unidentified, volatile or nonvolatile. During initial days, these were identified and controlled by qualitative tests like TLC and I.R or titration later with the advent of modern analytical chemistry more than $80 \%$ of drugs are now analyzed by chromatographic techniques for reporting of organic impurities. 


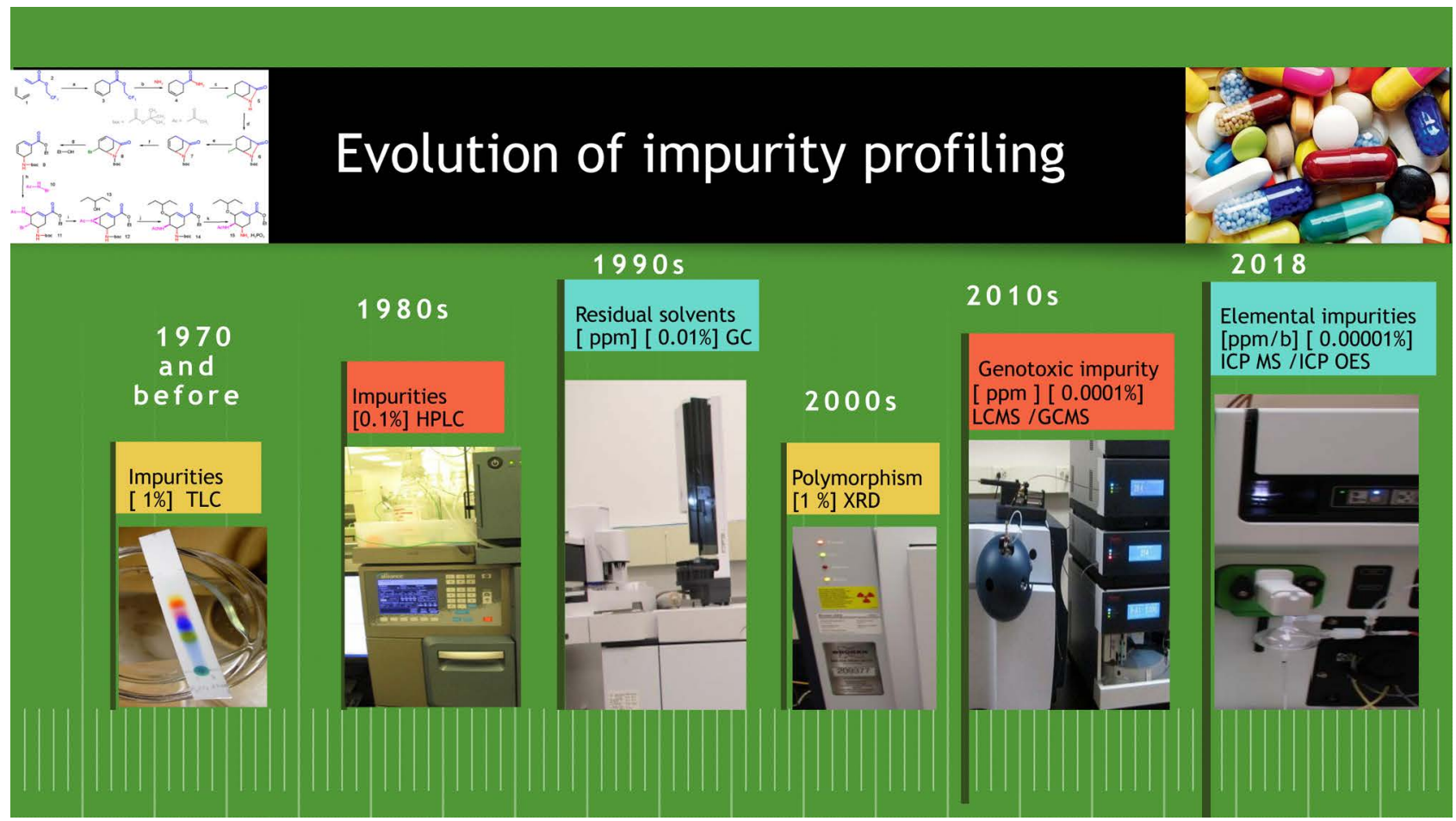

Figure 1. Evolution and Changes in Solid Oral Drug Products Impurity Profiling over the years.

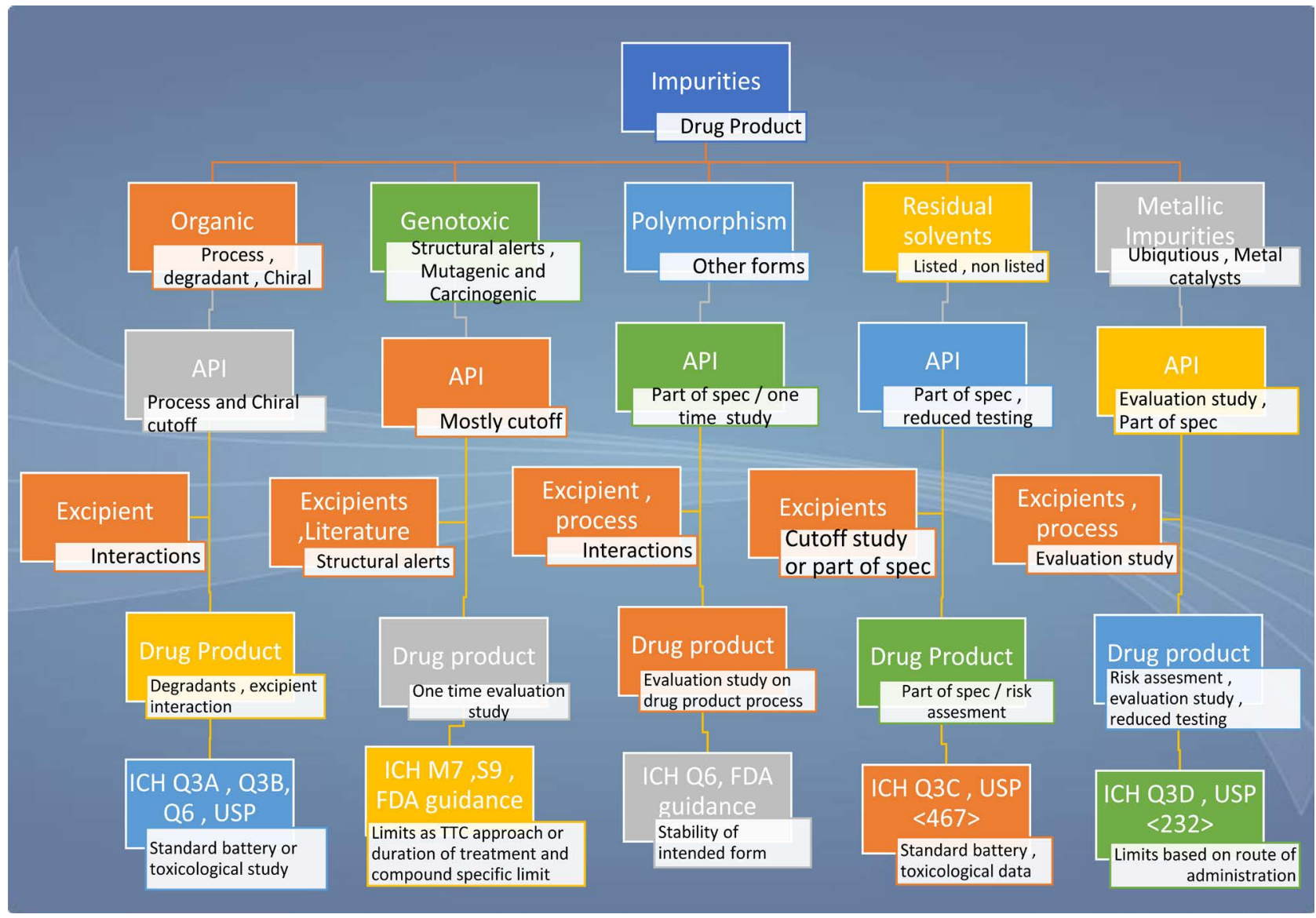

Figure 2. An overview of impurity profile. 
As per the GDUFA II guidance "ANDA Submissions-Refuse to Receive for Lack of Justification of Impurity Limits" during ANDA filing the justification for Organic impurities and their limits should be provided.

As per this guidance "FDA may RTR an ANDA for": failing to provide justification for proposed limits in drug substances and drug products for specified identified impurities that are above qualification thresholds; failing to provide justification for proposed limits for specified unidentified impurities that are above identification thresholds [11] [12]; and proposing limits for unspecified impurities (e.g., any unknown impurity) above identification thresholds. EP impurities are qualified for the levels in the EP monograph [13]. The information should be updated in the below tabular format in "eCTD section 3.2.S.4.5, 3.2.P.5.6 Justification of Specifications" Table 1 [14].

An Overview of organic impurities profiling is detailed in following illustration Figure 3.

Table 1. Impurity specification and justification.

Chemical Name Code \# MDD QT (\%) QT (TDI) Regulatory QT Threshold (\%)* Proposed AC (\%) Justification if proposed AC (\%) > Regulatory QT Threshold $(\%)^{* *}$

${ }^{*}$ Based on lower intake of impurity from QT (\%) or QT (TDI). If QT (TDI) is lower express as \%. ${ }^{*}$ Reference the section if supportive data is provided for justification.

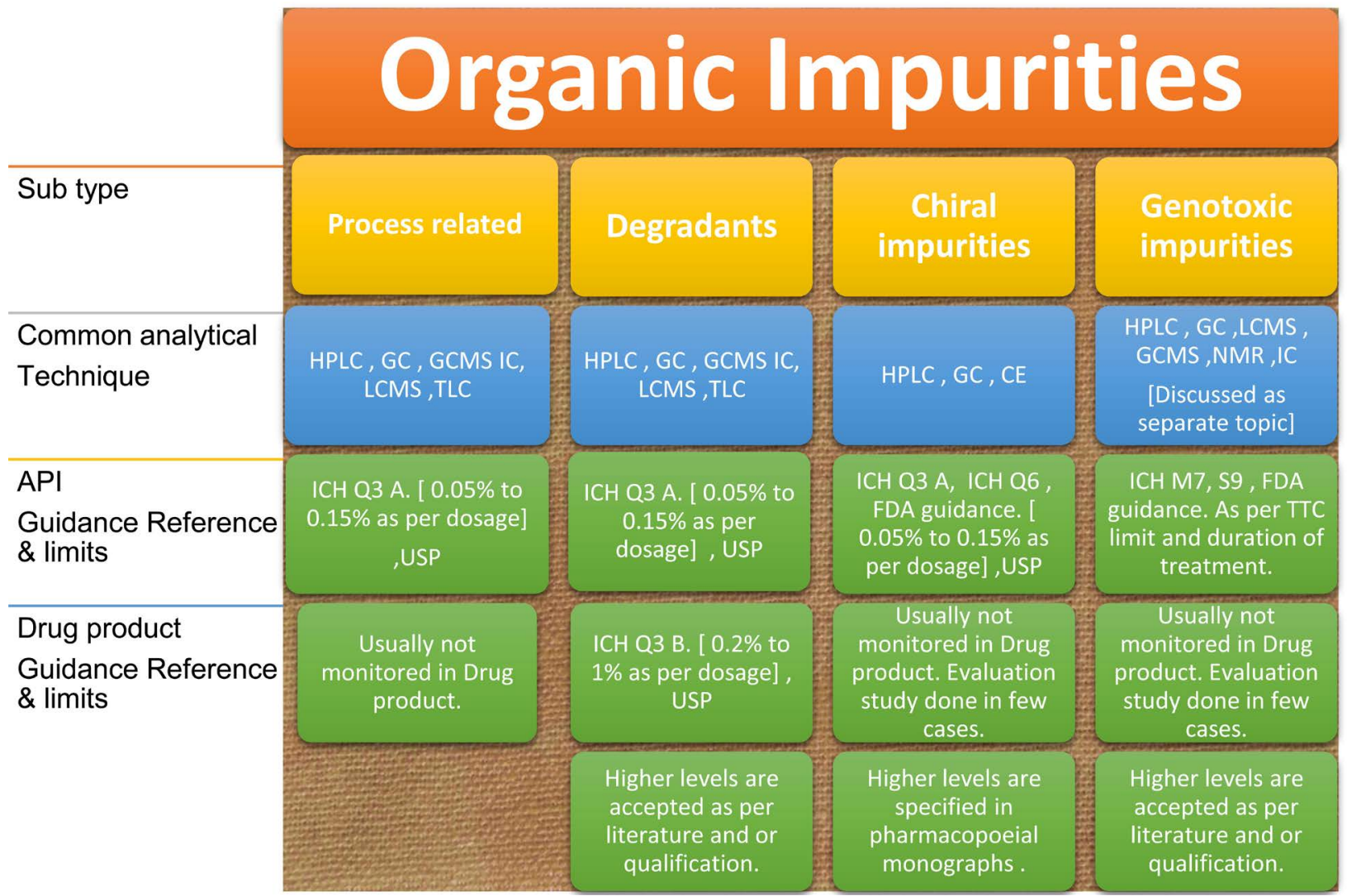

Figure 3. Organic impurities profiling in drug substance and drug products. 
Organic impurities are broadly classified into following categories

\subsection{Process Related Impurities}

Starting materials, Byproducts, Intermediates, Reagents, ligands, and catalysts. Impurities which are not increased in stability studies and not formed during forced degradation studies are considered as process related impurities.

Process related impurities are formed during the synthesis, purification, and storage of the drug substance. Most of these impurities will be expected during the synthesis based on knowledge on synthesis process, type of chemicals/reagents used, also these should be confirmed with stability studies [15] and degradation studies. The impurity selection should be cross verified or challenged by stress studies or force degradation studies [16].

In drug substances, impurities in final product specifications may be proposed based on the impurities detected in commercial process for setting better process control. Impurities which are above the qualification limits should be considered in specification with reporting and qualification thresholds based on its safe levels.

Where as in drug product demonstrating specificity (usually separation in chromatographic methods) for process related impurities with degradation impurities is requirement for a stability indicating method but there is no requirement to report process related impurities (as they are controlled in applicable raw materials) unless it is mentioned in respective pharmacopeial monograph.

\subsubsection{Analytical Techniques}

Advanced techniques for quantification of these impurities are High Performance Liquid Chromatography (HPLC), Ultra Performance Liquid Chromatography (UPLC)/Rapid resolution liquid chromatography (RRLC), Gas Chromatography (GC), Liquid chromatography-Mass Spectrometry (LCMS), Gas chromatography-Mass spectrometry (GC MS).

The limits are governed by ICH Q3A(R2) Impurities in New Drug Substances.

\subsubsection{Drug Substance Specification [11]}

Maximum daily dose: $\leq 2 \mathrm{~g}$ /day: $0.15 \%$ or $1.0 \mathrm{mg}$ per day intake (whichever is lower).

Maximum daily dose: $>2$ g/day: $0.05 \%$.

\subsubsection{Drug Product Specification}

Not considered for reporting unless it is mentioned in specific pharmacopeia monograph.

\subsection{Degradation Impurities/Products}

Forced degradation studies should be performed to identify potential degradation products, characterization of degradation product, stability of active molecule, to understand the degradation pathways of drug, rate of degradation, to develop a stability indicating analytical method and to define shelf life. 
Impurities which are increased during stability studies (Q1A) and/or formed during forced degradation studies are classified as degradation impurities/products.

The ICH Q1A guideline defines that the stress testing is designed to help "determine the intrinsic stability of the molecule by establishing degradation pathways in order to identify the likely degradation products and to validate the stability-indicating power of the analytical procedures used" [17].

The selection of conditions for degradation studies and levels of target degradation is practiced as per common industry practices and available peer reviewed publications. ICH guidance Q1B "Photostability testing of new drug substances and products" serves as a guidance document on photo stability [18].

WHO guidance document "Guideline on Submission of Documentation for Prequalification of Multi-source (Generic) Finished Pharmaceutical Products (FPPs) Used in the Treatment of HIV/AIDS, Malaria and Tuberculosis-GuideGenericSubmitDocFPPs_08_2005_WoAnnexes" [19] defines stress testing conditions in below Table 2.

The industry practices conditions include Hydrolysis at various $\mathrm{pH}$ (Oxidative, acid and base), photo degradation, and thermal degradation and humidity studies [20] [21]. Since the conditions varies for individual molecule, based on type of molecule and its physicochemical properties to attain degradation to a small extent, typically $10 \%-30 \%$ loss of active by assay [19]. The degradation should not be performed by exposing to harsher conditions to form secondary degradation products (Secondary degradation products are degradation products of degradation products and in most cases, are not observed during stability studies). Most of the common degradation pathways include chemical reactions such as, hydrolysis/dehydration, oxidation, isomerization/epimerization, rearrangements, decarboxylation, dimerization/polymerization, photolysis and transformation products involving reaction with excipients/salt forms. Some of the most common reactive impurities include aldehydes/reducing sugars, peroxides, nitrates, nitrites, metals and solvents [22]. In some cases, compounds with Halogens (Chlorine, Fluorine, Bromine) are considered as Structural alerts, carcinogenic substances which require genotoxicity evaluation to fix the specification.

Table 2. Typical degradation conditions.

\begin{tabular}{cc}
\hline Stress factor & Conditions \\
\hline Heat & $60^{\circ} \mathrm{C}$ \\
Humidity & $75 \% \mathrm{RH}$ or greater \\
Acid & $0.1 \mathrm{~N} \mathrm{HCl}$ \\
Base & $0.1 \mathrm{~N} \mathrm{NaOH}$ \\
Oxidative & $3 \% \mathrm{H}_{2} \mathrm{O}_{2}$ \\
Photolytic & Metal halide, $\mathrm{Hg} \mathrm{Xe} \mathrm{lamp,} \mathrm{or} \mathrm{UV-B/fluorescent}$ \\
Metal ions (optional) & $0.05 \mathrm{M} \mathrm{Fe}^{2+}$ or Cu ${ }^{2+}$ \\
\hline
\end{tabular}




\subsubsection{Analytical Techniques}

HPLC-PDA/UV detector, GC, LC MS, GC MS techniques shall be employed in identification of impurities with a validated analytical procedure [23].

As per the current requirements and recent deficiencies, forced degradation studies, peak purity studies and mass balance for individual impurity quantifying method should be performed. Mass balance is "the process of adding together the assay value and levels of degradation products to see how closely these add up to $100 \%$ of the initial value, with due consideration of the margin of analytical precision" [15].

Impurities found above identification threshold should be considered in evaluating impurity profile for drug substance/drug product.

\subsubsection{Drug Substance Specification [11]}

Maximum daily dose: $\leq 2 \mathrm{~g} /$ day: $0.15 \%$ or $1.0 \mathrm{mg}$ per day (whichever is lower)

Maximum daily dose: $>2 \mathrm{~g} /$ day: $0.05 \%$.

Performing individual excipient and drug compatibility studies is one of the recommended approaches to assess degradation pathways in Drug products. These studies should be designed as per the available literature and also known behavior for some excipients like povidone, silicon dioxide, Lactose, Polysorbate etc [24] [25].

Oxidative and photolytic conditions require special considerations to understand the mechanism of photodegradation and the potential for phototoxicity [26]. Photostability testing should be performed as per ICH Q1B as an integral part of stress testing [18].

\subsubsection{Drug Product Specification [12]}

Maximum daily dose: $<10 \mathrm{mg}: 1.0 \%$ or $50 \mu \mathrm{g}$ of TDI (Total daily intake) (whichever is lower)

Maximum daily dose: $10 \mathrm{mg}-100 \mathrm{mg}: 0.5 \%$ or $200 \mu \mathrm{g}$ of TDI (whichever is lower)

Maximum daily dose: $>100 \mathrm{mg}-2 \mathrm{~g}: 0.2 \%$ or $3 \mathrm{mg}$ of TDI (whichever is lower)

Maximum daily dose: $>2 \mathrm{~g}: 0.15 \%$.

High levels of impurities are acceptable with justification i.e. with literature and qualification.

\subsubsection{Most Common Related RTR Points}

- Impurities Specifications and table formats not as per guidelines to justify regulatory threshold.

\subsubsection{Common Deficiency Points}

- Method validation/Verifications not performed.

- In-house Method equivalency with monograph listed method.

- Mass balance for individual impurity methods is not demonstrated or not matching.

- System suitability not established for a validated method. 
- Specifications/Methods not as per Monograph.

- Specifications and justification for all potential impurities and unknown impurities in drug based on MDD.

- Missing Impurity characterization data.

- Tightening the limits as per trend.

\subsection{Chiral Impurities}

Enantiomers, non-superimposable mirror image stereoisomers with identical physico-chemical properties except that they rotate the plane of polarized light in opposite directions and by equal amounts [27].

If one of the enantiomer is considered as active ingredient, then the other may/shall be considered as impurity/undesired.

In earlier days, there was not much focus on individual enantiomers and were not well studied or characterized due to limitation of analytical techniques. Current advanced techniques allow to define, study completely, separate and quantify stereoisomeric impurities [28].

If applicable, stereoisomeric purity of starting material should be studied with a well resolved and validated stereoselective test procedure and possible formation of stereogenic center in individual synthetic process details and controls should be established [29].

For a drug substance stability indicating method, it is required to perform forced degradation studies for chiral impurity methods and confirm its formation. If the chiral impurity increases in stability studies, then it should be monitored in drug product.

\subsubsection{Analytical Techniques}

Advanced analytical techniques used in chiral separation are Gas Chromatography (GC), High Performance Liquid Chromatography (HPLC), UHPLC [30], Capillary Electrophoresis (CE) and liquid phase Nuclear Magnetic Resonance Spectroscopy (NMR). Recent trend of emergence of Polysaccharide derived chiral stationary phases (CSPs) have been recognized as the most powerful packing materials for the chromatographic separation of enantiomers in analytical and preparative applications due to their broad application field and their remarkable loading capacity [31]-[40], a wide variety of solvents both polar and non-polar can be used to achieve desirable separations.

Scientists were able to achieve satisfactory resolution [ $>1.5]$ and system suitability criterion theoretical [ $>5000]$ and tailing factor is between 0.8 and 2.0 in the analysis of bicalutamide and thalidomide enantiomers using variety of solvents [41]:

1) Methanol: Hexanes: THF (30:50:20 $v / v / v)$

2) MDC: Methanol: $n$-hexane (40:20:40 $v / v / v)$

3) Ethyl Acetate: Methanol: n-Hexane (30:55:15 $v / v / v)$

4) MTBE: n-Hexane: Methanol (50:10:40 $v / v / v)$.

The system suitability criterion and the quantification for Bicalutamide and 
Thalidomide enantiomers is comfortably achieved across all the four mobile phase compositions which is presented in Figure 4.

\subsubsection{Drug Substance Specification}

Maximum daily dose: $\leq 2 \mathrm{~g} /$ day: $0.15 \%$ or $1.0 \mathrm{mg}$ per day (whichever is lower).

Maximum daily dose: $>2 \mathrm{~g} /$ day: $0.05 \%$.

In most of the cases chiral impurities are controlled in drug substance but in some cases control of chiral impurities and confirmation of unacceptable change in stereochemical purity or ratio of the active substance occurs in drug product shelf life should be established with a validated analytical method [42].

\subsubsection{Drug Product Specification}

In general, not included in specification, but in some cases evaluation may be required.

\subsubsection{Most Common Related RTR Points}

- Limits/Controlling for chiral impurities not established.

\subsubsection{Common Deficiency Points}

- System suitability not established for methods.

- Standards used which are with less potency.

- Optical purity not established.

- Method/formulation processes not understood properly to verify formation of other possible chiral form which may arise from reagents and chemicals used in the process.

- Specifications for stereo isomers/geometric isomers wherever possible.

- Chiral assay test based on the amount of chiral impurity to demonstrate racemization on storage.

- Establish LOD, LOQ limits with a validated method for all specified impurities.

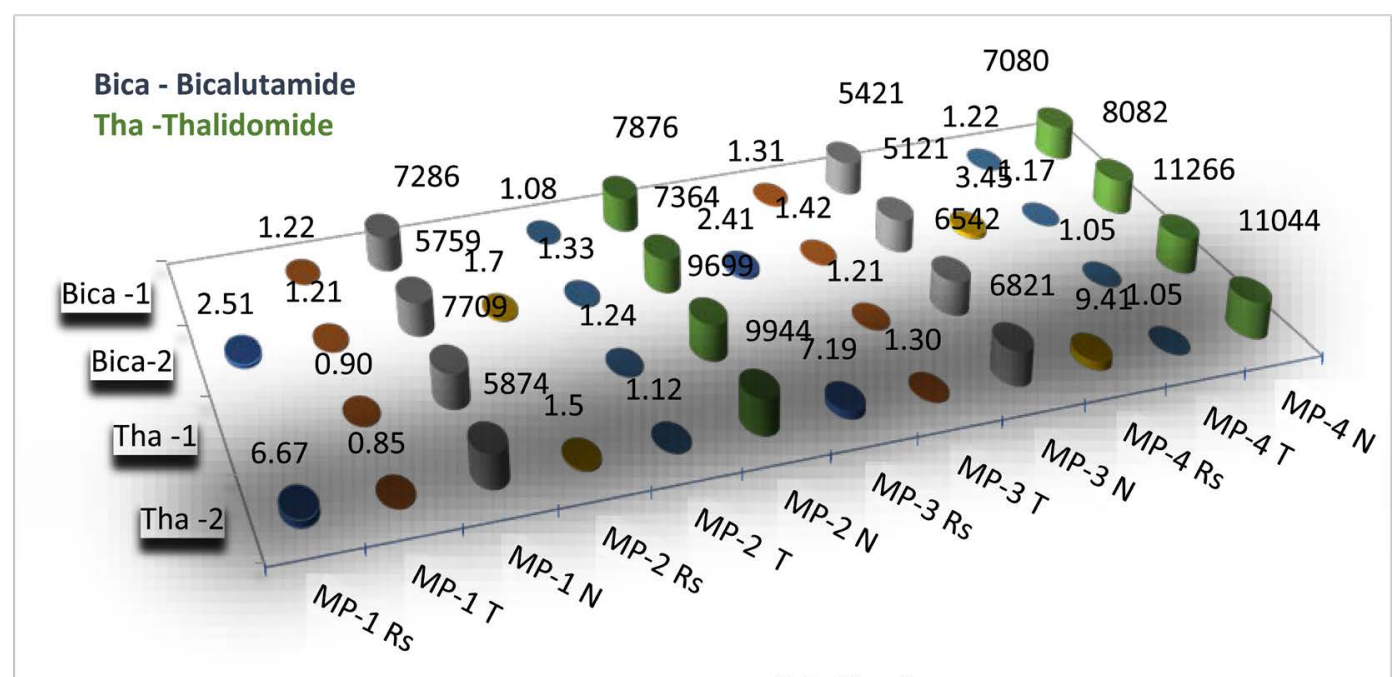

MP-Mobile Phase

Rs -Resolution

T-Tailing Factor N-Theorotical plates

Figure 4. Comparison of performance parameters across mobile phases [41]. 
- Missing Impurity characterization data.

- Tightening the limits as per trend.

\section{Inorganic Impurities}

Inorganic impurities can result from the manufacturing process. They are normally known and identified and include Reagents, Ligands, and Catalysts, Heavy metals or Other residual materials, Inorganic salts, Other materials (e.g. filter aids, charcoal) [1]. Heavy metals test by color comparison is a part of USP from 1904, due to numerous events on health of patient population the controls on metallic impurities is effective and in full control from Jan. $1^{\text {st }} 2018$ across all the major regulatory authorities.

\subsection{Elemental Impurities}

Based on decades of studies, knowledge of toxicological effects and to improve safety and efficacy of drugs, current requirements changed and replaced nonspecific heavy metal tests with specific quantifying techniques [43] and specifications [44] were included. The classical color comparison test in Pharmacopeia is deficient in detecting few metallic impurities. The standard solutions of all the metals are also of different solubilities due to the variable levels of reactivities of metals with sulfide ion, which also creates a problem in visual comparison with lead standard as shown in Figure 5 [45].

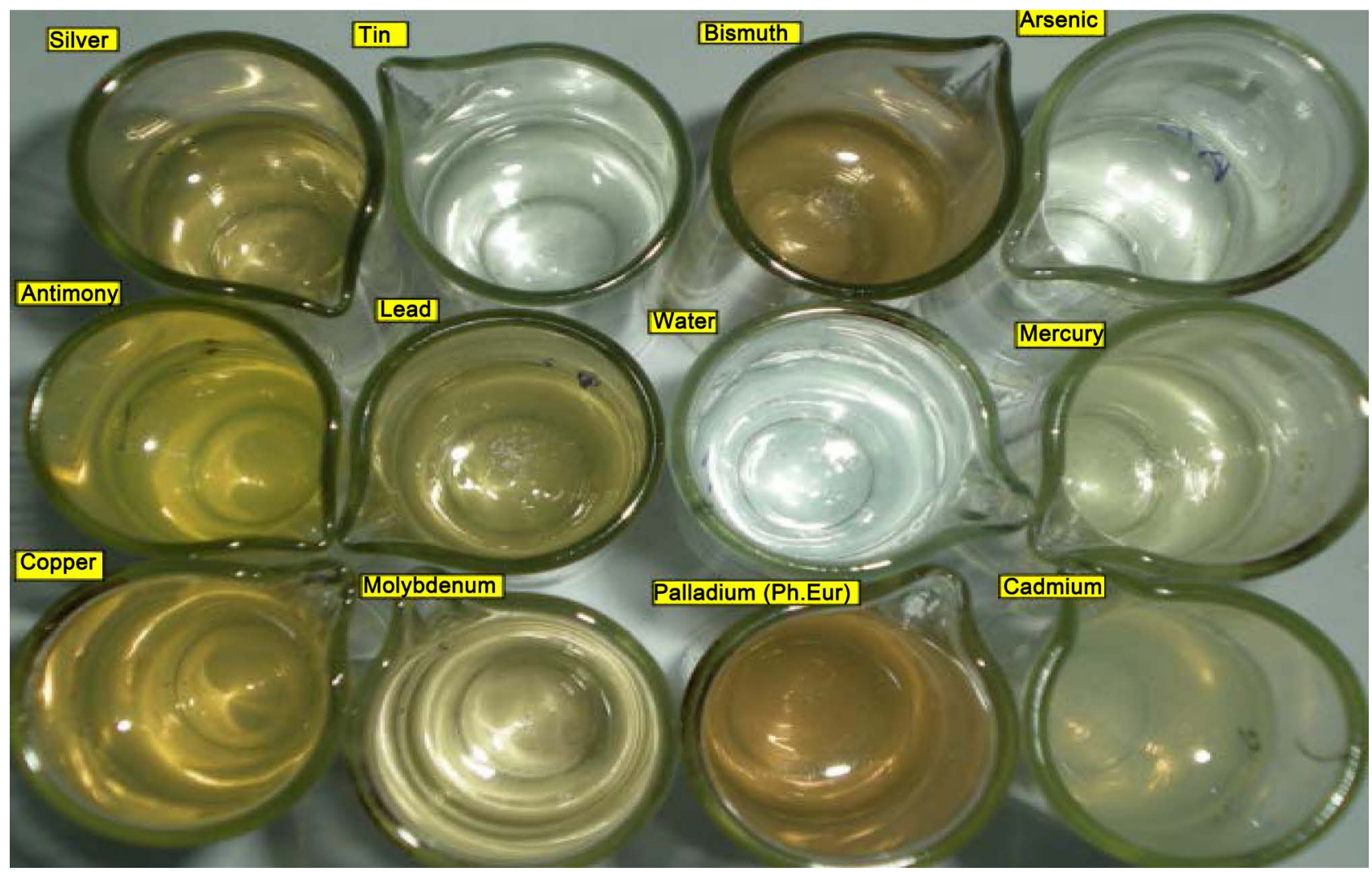

Figure 5. Erroneous reporting of heavy metals as per USP $<231>$ heavy metals [45]. 
Process catalysts and environmental contaminants may be present in drug substances, excipients, or drug products. These impurities may occur naturally, be added intentionally, or be introduced inadvertently. As elemental impurities do not provide any therapeutic benefit to the patient, besides continuous exposure might be toxic (as the toxicity is related to its extend of exposure), so their toxic levels in the drug product should be controlled within acceptable limits [46].

\subsection{Risk Assessment}

Elemental impurities are classified into 4 categories [1,2A, 2B and 3] based on the route of administration, limits and requirements for control varies. Risk assessment should be done for elements: $\mathrm{Cd}, \mathrm{Pb}, \mathrm{As}, \mathrm{Hg}, \mathrm{Co}, \mathrm{V}, \mathrm{Ni}, \mathrm{Tl}, \mathrm{Au}, \mathrm{Pf}, \mathrm{Ir}$, Os, Ph, Ru, Se, Ag, Pt, Li, Sb, Ba, Mo, Cu, Sn, Cr as per ICH Q3 D [47] and USP $<232>$ based on potential source, Evaluation of toxicity data with respect to route of administration, Establishment of a Permitted Daily Exposure (PDE) and Justification for higher levels than established PDE.

In drug product risk evaluation, potential sources of elemental impurities should be considered are elements intentionally added such as process catalysts, elements potentially present in the materials used to prepare the drug product, and elements potentially introduced from manufacturing equipment or container closure systems [46] [47].

Based on risk assessment, it should be concluded to include testing of elemental impurities in routine testing for components of drug product or for drug product i.e. if elemental impurities are controlled in raw material (active and inactive) as part of routine testing, in drug product routine testing may not be required. ICH Q3 D defines different options (Option 1, Option 2a, Option 2b and Option 3) for risk assessment and control strategy (If batch analysis results are consistently less than $30 \%$ of the PDE then additional controls are not required).

As per FDA guidance, Elemental impurity risk assessment for ANDA's filed and approved before Jan $1^{\text {st }} 2018$ should be updated in annual updates, if any process changes are needed to be made to meet the Elemental impurities requirements they should follow appropriate regulatory filing strategy and for ANDA's to be filed, it is a requirement to be considered for filing [46]. An overview of elemental impurities as per ICH Q3D to consider for risk assessment, presented below Figure 6.

\subsection{Specifications}

ICH Q3 D classifies metallic impurities into Class 1, 2A, 2B and 3 with decreasing level of toxicity.

The specifications and class of impurities to be controlled varies on route of administration.

\subsection{Analysis Techniques}

In general, typical analytical techniques followed for elemental impurities analysis 


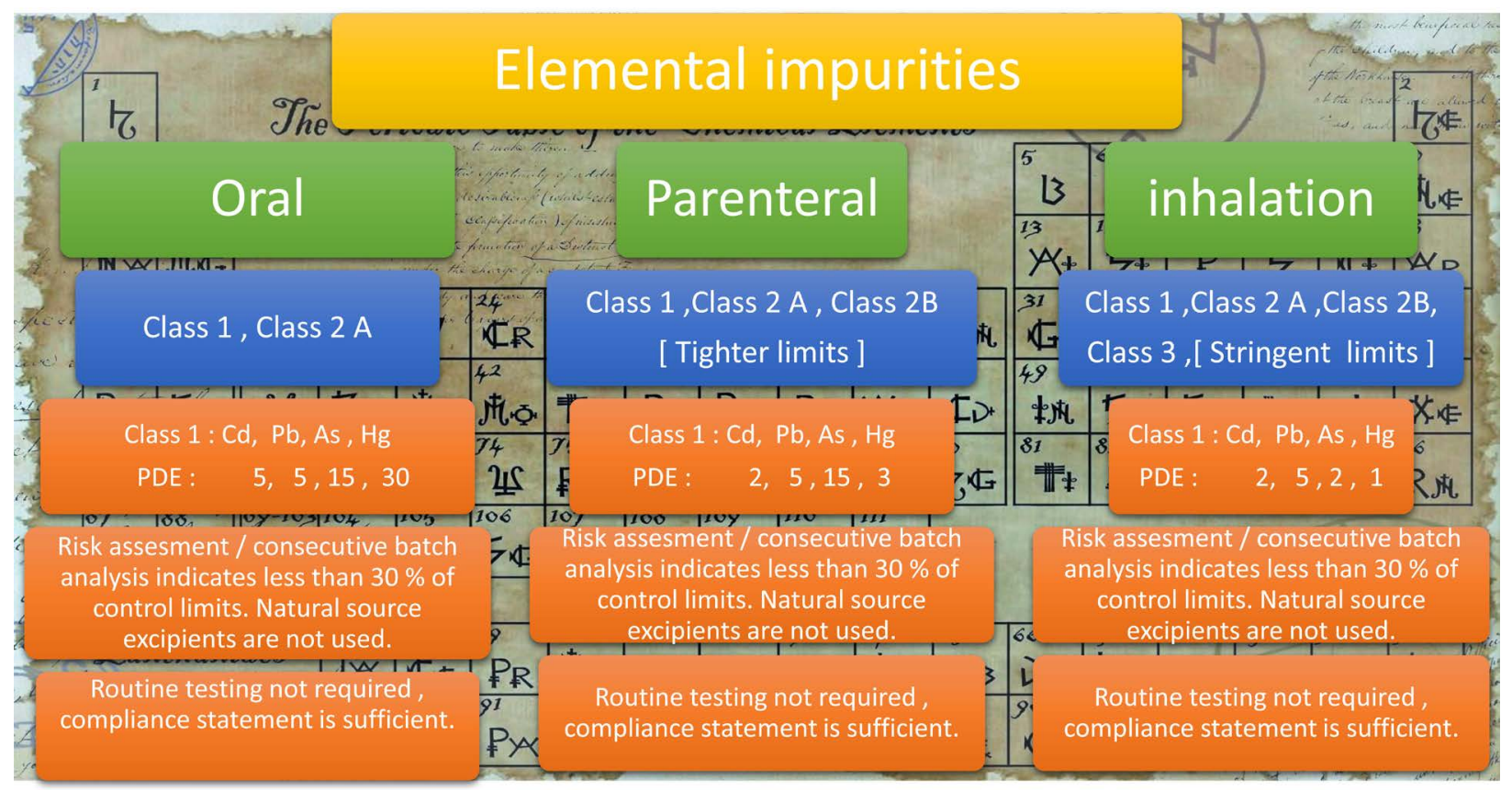

Figure 6. Overview of elemental impurities.

are ICP, ICP-MS. These can be analyzed with the method listed in USP <233>, if alternate methods are used those are to be validated as per ICH Q2, USP $<1225>$. There are few alternate analytical methods available and reported in research articles [45].

\subsection{Most Common Related RTR Points}

- It is an emerging topic.

\subsection{Common Deficiency Points}

- Quality risk assessment complete documentation not provided.

- Complete assessment report and related documents for ANDA's filed before Jan $1^{\text {st }} 2018$.

- Excipient batch analysis data.

- Consistency between batches which contains excipients like Sodium bicarbonate, Calcium carbonate.

- More batch data is required when natural source excipients like sodium carbonate and sodium bicarbonate are used.

\section{Residual Solvents}

Residual solvents are organic liquids used as vehicles for the preparation of solutions or suspensions in the synthesis of a drug substance or excipients or in the preparation of drug product.

The residual solvents used in each stage of manufacturing process of Drug substance or excipient can either be analyzed and exhibited that they meet the ICH Q3C [48], USP <467> requirements [49] or they can be analyzed in final 
stage, in either cases they should be analyzed with validated method or method specified in USP <467> [49].

The residual solvents with known limits and toxicity details with respect to their class were listed in ICH Q3C, their specifications in final product should be maintained to the possible lowest extent. (The acceptance limits should be based on ICH Q3C, USP $<467>$ ). If non listed solvents are used they should be controlled as per respective solvent toxicological limit.

In recent revision of ICH Q3C Triethylamine is added as Class-3 solvent with PDE limit (62.5 mg/Day) and limit for Methyl isobutylketone is revised (Class-2) PDE limit (45 mg/day) as per the new toxicological data.

\subsection{Analytical Techniques}

Usually GC and GC-HS methods are employed and in some cases HPLC, IC and other techniques are employed. If residual solvent method is specified in a monograph, the same can be followed or method specified in USP $<467>$ can be used. USP $<467>$ does not specify a method for class- 3 solvents but FDA cites to control and report class 3 solvents also with a validated method. Usually residual solvents analysis is done by gas chromatography technique, but some of the class-3 solvents like Acetic acid, triethylamine, formic acid etc might not be accurately quantified with Head space sampler Gas chromatography, even in auto liquid sampler acetic acid may elute but the improper peak shape may pose problems in meeting the analytical method validation criterion (Figure 7) [Chromatogram eluted by GC -Auto Liquid sampler method on FID detector $-250^{\circ} \mathrm{C}$ using DB WAX column $-30 \mathrm{~m} \times 0.53 \mathrm{~mm} \times 1.0 \mu \mathrm{m}$, Inlet temperature $240^{\circ} \mathrm{C}$, Carrier gas-Nitrogen $3.0 \mathrm{~mL} / \mathrm{min}$, Split ratio 1:5 and Makeup gas: 25 $\mathrm{mL} / \mathrm{min}$ Nitrogen]. These solvents may be quantified using other techniques like High performance liquid chromatography (Figure 8) [Chromatogram eluted by HPLC, Reverse phase Grace Altima C18, $150 \times 4.6 \mathrm{~mm}, 3 \mu \mathrm{m}$ at $210 \mathrm{~nm}, 0.8$ $\mathrm{mL} / \mathrm{min}$ gradient flow; Mobile phase-A: $0.01 \mathrm{M}$ sodium phosphate monohydrate

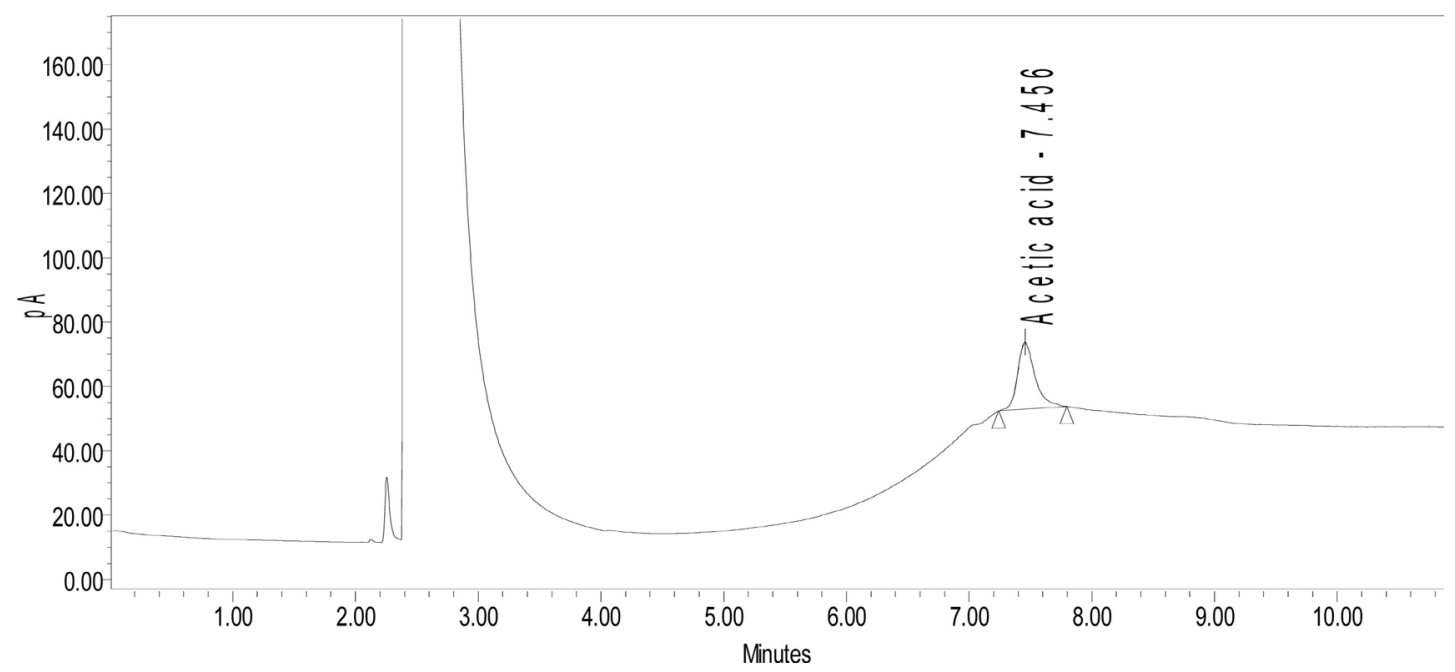

Figure 7. Acetic Acid content by GC. 


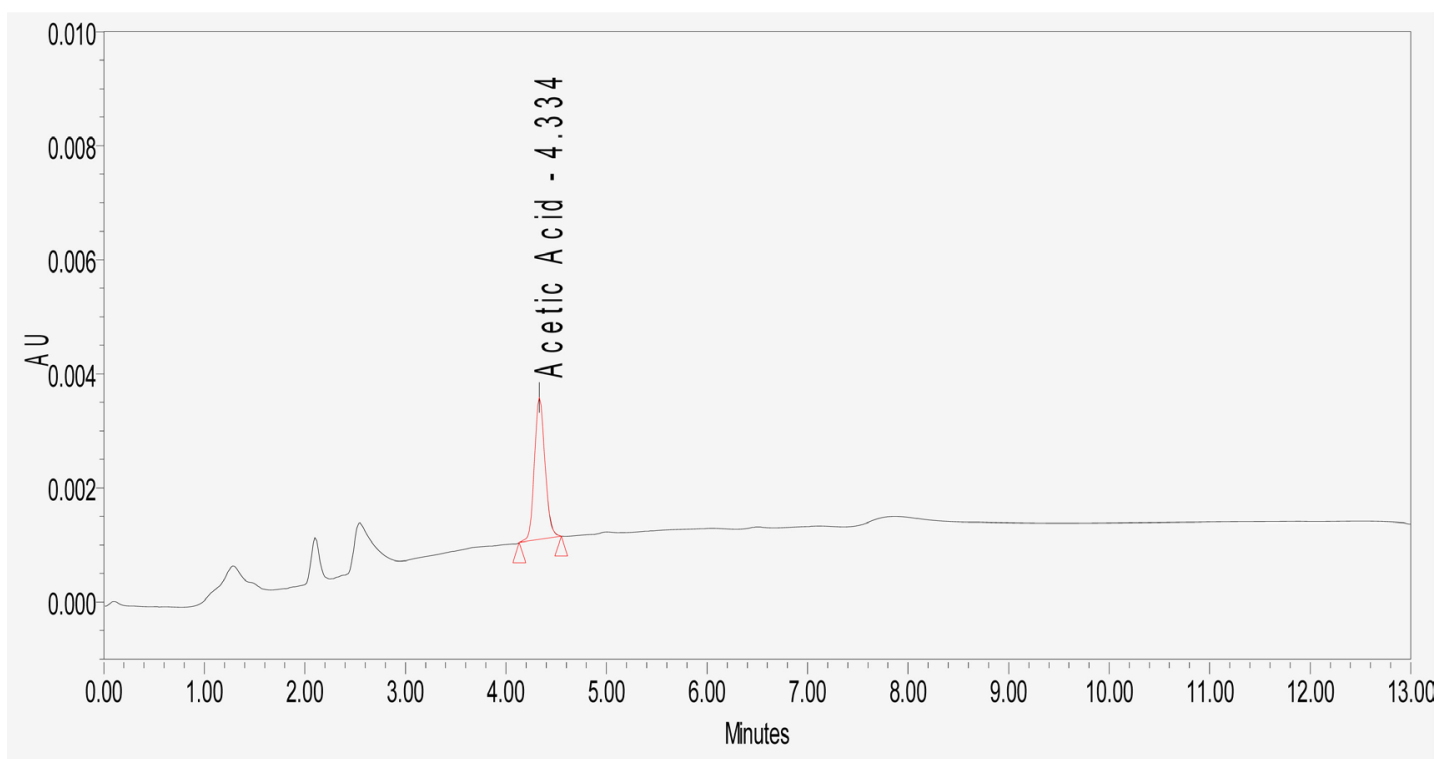

Figure 8. Acetic Acid content by HPLC.

pH 2.0, Mobile phase-B Acetonitrile: Water $-80: 20 \mathrm{v} / \mathrm{v}$ ], Ion exchange chromatography. Some of the drug substances manufacturing process use non-listed solvents, for those solvents there may be published research articles available or USP <467> methods can be modified. Scientists achieved separation and Quantification of Cyclopropylamine, Diethylamine and Triethylamine in Active Pharmaceutical Ingredients [50].

\subsection{Drug Substance Specifications}

As per ICH Q3C classification.

\subsection{Drug Product Specification}

As the residual solvents limits were set as per the guidelines and quantified in individual excipient and drug substance, testing for solvents in drug product is not required. Compliance may be demonstrated through comprehensive risk assessment based on quantified results of residual solvents present in individual drug substance and excipients [51].

If residual solvents are used in the manufacturing process of drug product, they should be quantified with validated analytical methods with ICH Q3C limits.

If a non-listed solvent is used in the process, then justification for limits proposed, control strategy and toxicity evaluation should be presented/executed.

\subsection{Most Common Related RTR Points/DMF Initial Review Points}

- Specification not included for Residual solvents used in drug product formulation.

- Improper justification for residual solvents with limits not as per ICH Q3C, USP $<467>$ and their effect on human drug quality. 


\subsection{Common Deficiency Points}

- All residual solvents not included in specification.

- Drug substance residual solvents specification in line with ICH Q3C, USP $<467>$ and DMF.

- Excipient residual solvents not considered for assessment/ specification.

- Excipient residual solvents methods not validated/ verified.

- Tighten the solvents limits based on trend data.

- Establish LOQ, LOD limits for all residual solvents and LOQ limits should be at least $50 \%$ of specification.

- Unidentified peaks in samples which are to be analyzed with validated method.

\section{Polymorphic Forms/Impurities}

Polymorphism evaluation shall be used for estimation of amorphous and crystalline of qualitative and quantitative as per USP <941>, ICH Q6A and FDA guidance "ANDAs: Pharmaceutical Solid Polymorphism" [52] [53].

As the physical properties of a raw material may change with polymorphic form, affect on manufacture process of the drug substance and the drug product, as well as on drug product stability, dissolution, and bioavailability, the type and extent of characterization and release testing performed on the co-crystal should be sufficient to ensure the identity, strength, quality, and purity of the API(s) and drug product stability [54] [55] [56]. Any polymorphic form other than desired polymorphic form will be considered as impurity [57].

Based on the process and evaluation done by drug substance manufacturer polymorphic form will be confirmed.

In drug product, drug substance polymorphic form should not change due to drug product manufacturing process or due to excipients or due to storage over a period of time. Drug product initial samples and stability samples should be evaluated to prove the polymorphic form stability throughout the shelf life [53]. Information on different polymorphs of drug substances is generally referred in respective patents.

\subsection{Analytical Techniques}

Common analytical technique used is polymorphism by $\mathrm{p}$ X-ray diffraction. Method adopted should be specific to desired crystalline form of the compound based on drug substance manufacturing process. Method should be validated as per USP $<1225>$ [23].

In some cases XRD pattern of different polymorphic forms might be similar, then other techniques like Thermal analysis (DSC) or Raman spectroscopy should be used.

Omeprazole form A and form B can be identified by different techniques (e.g.: XRPD, FTIR and Raman spectrometer) among them Raman spectrometer technique is more specific as it can also identify amorphous forms along with crys- 
talline forms. Polymorphism evaluation in Omeprazole is reported by Raman spectroscopy that Omeprazole ${ }^{\mathrm{TM}}$ form $\mathrm{A}$ is easily characterized by the absence of a band at $1364 \mathrm{~cm}^{-1}$, which is observed in Omeprazole form $\mathrm{B}$, and by the ratio of the relative intensities of 840.87 and $821.95 \mathrm{~cm}^{-1}$ bands.

The ratio (intensity of $840.87 \mathrm{~cm}^{-1}$ band/intensity of $821.95 \mathrm{~cm}^{-1}$ band) is $<1$ for Omeprazole Form A, while the ratio is $>1$ for Omeprazole form B (Figure 9) [58].

Fexofenadine hydrochloride form identification can be done by DSC (Melting point: $142^{\circ} \mathrm{C}$ is Form-VIII, Melting point: $155.35^{\circ} \mathrm{C}$ is Form X, Melting point: $138.61^{\circ} \mathrm{C}$ is Form IX) [59].

Very few monographs in USP have the specification for identification by polymorphism (e.g.: Colloidal Activated Attapulgite, Indomethacin).

\subsection{Common Deficiency Points/Information Requests for}

- Include test for polymorphism in specification.

- Polymorphic stability of the molecule in drug substance and drug product to be demonstrated.

- Demonstrate polymorphic stability throughout the drug substance and drug product stability.

- Evaluate polymorphic stability effect on dissolution / drug product blending and/or unit operations.

- Demonstrate possibility of existence of other forms in drug substance.

- Validation of analytical technique used for polymorphic study.

\section{Genotoxic Impurities (Mutagenic and Carcinogenic Potential)}

Genotoxic impurities are DNA reactive substances that have a potential to directly cause DNA damage when present at low levels leading to mutations and

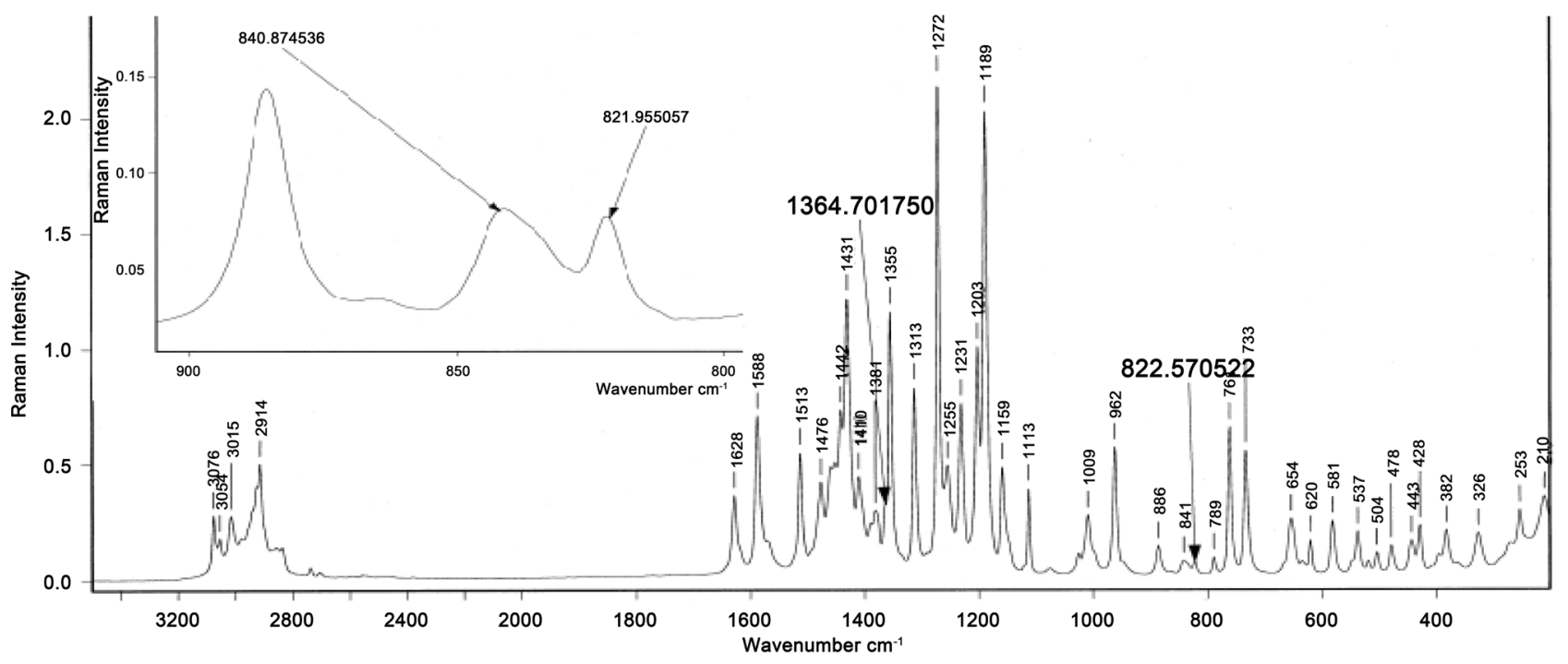

Figure 9. Omeprazole Form-B polymorphism by Raman spectroscopy. 
therefore, potentially causing cancer. This type of mutagenic carcinogen is usually detected in a bacterial reverse mutation (mutagenicity) assay. Structure-based assessments are useful for predicting bacterial mutagenicity outcomes based upon the established knowledge. There are a variety of approaches to conduct this evaluation, including a review of the available literature and/or computational toxicology assessment [60].

ICH M7 definition of genotoxic impurities does not apply to drug substances and drug products intended for advanced cancer indications as defined in the scope of ICH S9 [60] [61] [62]. Additionally, there may be some cases where a drug substance intended for other indications is itself genotoxic at therapeutic concentrations and may be expected to be associated with an increased cancer risk. Exposure to a mutagenic impurity in these cases would not significantly add to the cancer risk of the drug substance. Therefore, impurities could be controlled at acceptable levels for non-mutagenic impurities [63].

Impurities below the identification limit with structural alerts (e.g. N-nitroso-, and alkyl-azoxy compounds), and potential impurities should be considered for genotoxic/mutagenic evaluation [64]. This risk-based assessment of individual synthetic process should be performed by considering starting material/reagents used in the process and intermediate/byproducts formed during the synthesis process [63]. Suitability of the proposed control strategy can be supported with information about any mutagenic impurities formed or purged in the manufacturing steps between the proposed starting material and the drug substance, or that are controlled in the specification of the proposed starting material [64].

\subsection{Analytical Techniques}

LCMS, GC MS are normally used for genotoxic impurity detection and quantification to achieve desired limit of quantification. HPLC, UPLC and GC methods may be used in some cases.

\subsection{Threshold of Toxicological Concern (TTC)}

Threshold of Toxicological Concern (TTC) concept was developed to define an acceptable intake for any unstudied chemical that poses a negligible risk of carcinogenicity or other toxic effects. The methods upon which the TTC is based are generally considered to be very conservative since they involve a simple linear extrapolation from the dose giving a 50\% tumor incidence (TD50) to 1 in $10^{6}$ incidence cases, using TD50 data for the most sensitive species and most sensitive site of tumor induction [60].

The concentration limits in ppm of genotoxic impurity in drug substance derived from the threshold of toxicological concern (TTC) can be calculated based on the expected daily dose to the patient using equation [60] [65].

$$
\text { Concentration limit }(\mathrm{ppm})=\mathrm{TTC}[\mu \mathrm{g} / \text { day }] / \text { dose }(\mathrm{g} / \text { day })
$$

An overview of the genotoxic impurities guidance is presented in Figure 10. 
ICH guidance M7 classifies possible genotoxic impurities into 5 classes, which is presented in Table 3 below.

Standard risk assessments of known carcinogens assume that cancer risk increases as a function of cumulative dose. Thus, cancer risk of a continuous low dose over a lifetime would be equivalent to the cancer risk associated with an identical cumulative exposure averaged over a shorter duration.

ICH M7 defines limits for single impurity and for total of two or more impurities as per duration of treatment as per below Figure 11 limits [60].

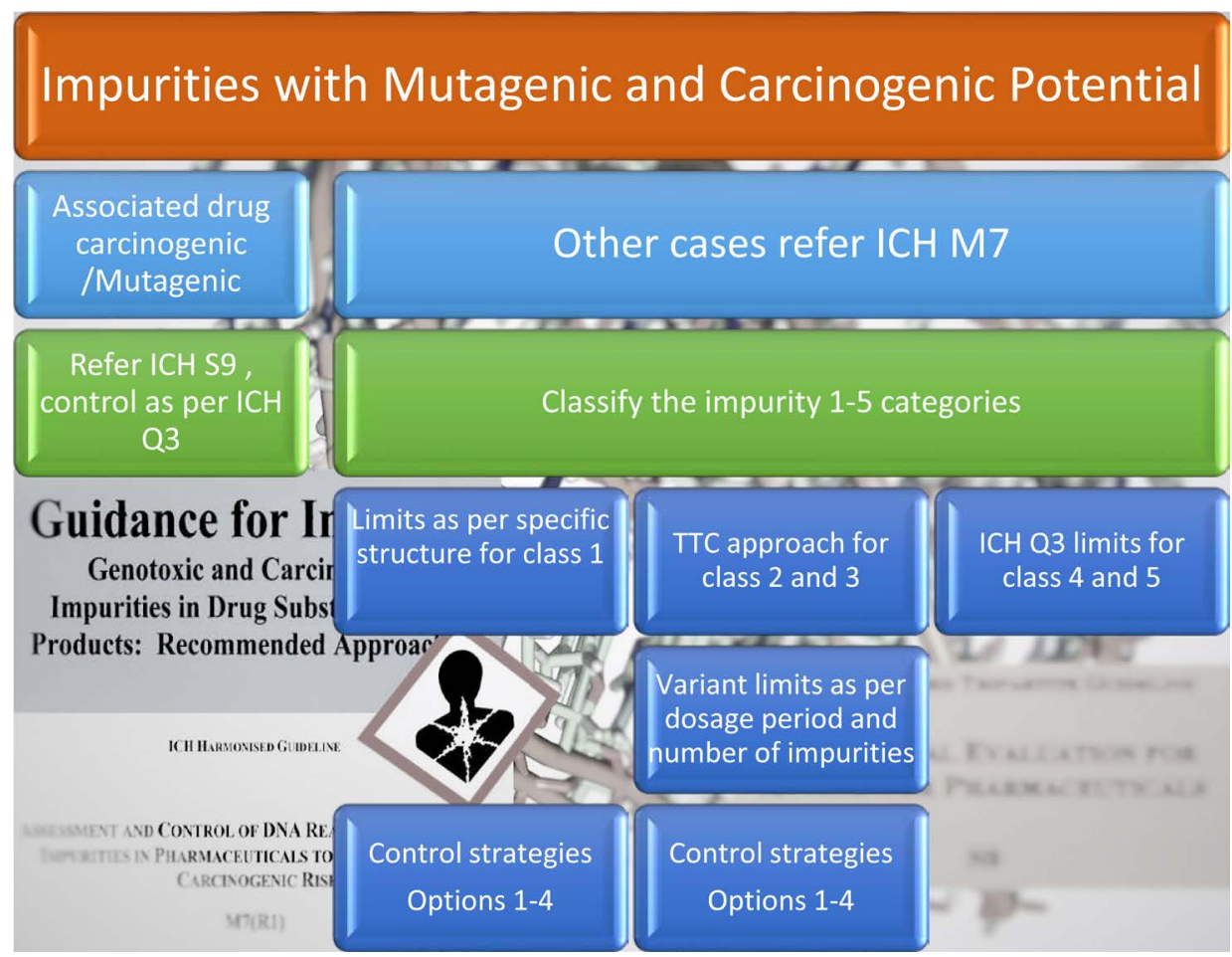

Figure 10. An overview of genotoxic impurities.

Table 3. Genotoxic impurities as per ICH M7 classified into five classes [60].

\begin{tabular}{cll}
\hline Class & \multicolumn{1}{c}{ Definition } & Proposed action for control (details in Section 7 and 8) \\
\hline $\mathbf{1}$ & Known mutagenic carcinogens & Control at or below compound-specific acceptable limit \\
$\mathbf{2}$ & $\begin{array}{l}\text { Known mutagens with unknown carcinogenic potential (bacterial } \\
\text { mutagenicity positive*, no rodent carcinogenicity data) }\end{array}$ & Control at or below acceptable limits (appropriate TTC) \\
$\mathbf{3}$ & $\begin{array}{l}\text { Alerting structure, unrelated to the structure of the drug substance; } \\
\text { no mutagenicity data }\end{array}$ & $\begin{array}{l}\text { Control at or below acceptable limits (appropriate TTC) or } \\
\text { conduct bacterial mutagenicity assay; }\end{array}$ \\
& $\begin{array}{l}\text { Alerting structure, same alert in drug substance or compounds related to the } \\
\text { drug substance (e.g., process intermediates) which have been tested and are } \\
\text { non-mutagenic }\end{array}$ & $\begin{array}{l}\text { Treat as non-mutagenic impurity } \\
\text { If non } 2\end{array}$ \\
$\begin{array}{l}\mathbf{5} \\
\text { No structural alerts, or alerting structure with sufficient data to demonstrate } \\
\text { lack of mutagenicity or carcinogenicity }\end{array}$ & Treat as non-mutagenic impurity
\end{tabular}

${ }^{*}$ Or other relevant positive mutagenicity data indicative of DNA-reactivity related induction of gene mutations (e.g., positive findings in in vivo gene mutation studies). 


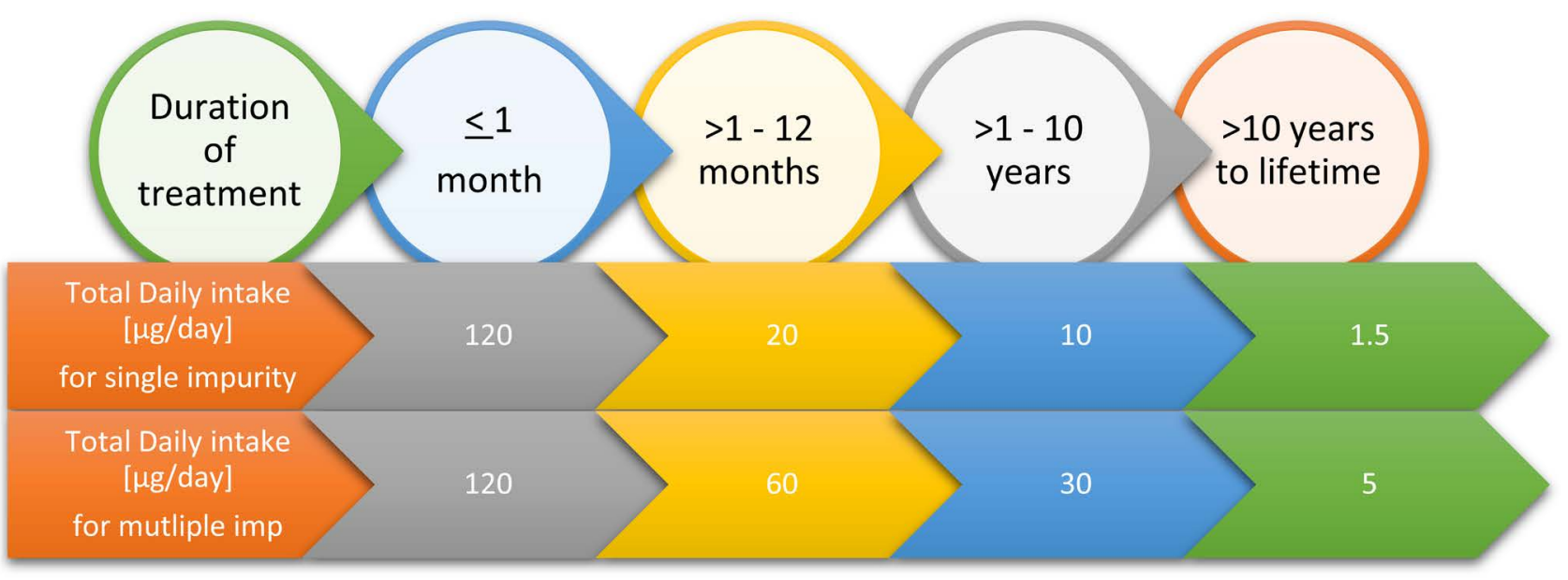

Figure 11. Limits for class 2 and class 3 impurities as per duration of treatment.

ICH M7 defines different control strategies with Options 1 - 5. Option 3 allows manufacturers to avoid release testing if the impurities are less than $30 \%$ of control in few batches.

Pharmacopeia's are embracing this concept slowly however regulatory authorities are watchful and issuing deficiency to control the impurities. Bromochloropropionphenone impurity in Bupropion Hydrochloride, USP specifies this impurity at NMT $0.1 \%$ but as per literature impurity should be controlled at 4 ppm, FDA has cited this as a deficiency for the filers.

\subsection{Specifications}

As per ICH M7 and S9 or respective impurity toxicological limits.

\subsection{Common Deficiency Points}

- Possible limits not demonstrated: Genotoxicology study of a compound not demonstrated.

- USP limits not adequate as compound has structural alerts.

- Study or cut off for all possible structural alerts not demonstrated.

- Limits should be tightened as per toxicological limits.

- One-time study not adequate for genotoxic impurity and to be included in specification.

- Spike \& purge studies data to be presented for control option selected.

\section{Acknowledgements}

Authors thank Sciegen management and Dr. Subbarao Devarakonda, Shailaja Pailla, Priyanka Bodireddy and Anitha Yerikareddy for their help, review and inputs in writing this review article.

\section{References}

[1] (1086) USP General Chapter. 
[2] CDER (2018) Draft Guidance on Good ANDA Submission Practices.

[3] GPhA (2014) Enhancing ANDA Submissions: Working towards First Cycle Approval. A GPhA White Paper.

[4] CDER and Office of Generic Drugs (2014) Draft Guidance, ANDA Submissions-Amendments and Easily Correctable Deficiencies.

[5] CDER (2018) Manual of Policies and Procedures. Establishing Impurity Acceptance Criteria as Part of Specifications for NDAs, ANDAs, and BLAs Based on Clinical Relevance, Effective Date: January 18, 2018.

[6] CDER (2003) Guideline on INDs for Phase 2 and Phase 3 Studies-Chemistry, Manufacturing, and Controls Information.

[7] Srinivasan, A. and Iser, R. (2018) FDA Perspectives: Common Deficiencies in Abbreviated New Drug Applications: Part 1: Drug Substance. Pharmaceutical Technology, 34, 50-59.

[8] WHO/POT (2016) Guidance Document on Guidance on Amendments to an Active Pharmaceutical Ingredient Master File (APIMF) or (FPP) or Prequalified Active Pharmaceutical Ingredient (API).

[9] UN (2001) Drug Characterization and Impurity Profiling-Background and Concepts. United Nations International Drug Control Programme, New York.

[10] Keitel, S. (2006) Impurity Profiles in Active Pharmaceutical Ingredients. EU/Swissmedic GMP Workshop, Beijing University, 20-22 September 2006.

[11] ICH Q3A Impurities in New Drug Substances.

[12] ICH Q3B Impurities in New Drug Products.

[13] EDQM (2015) Technical Guide for the Elaboration of Monographs. 7th Edition.

[14] CDER and Office of Generic Drugs (2016) Summary Tables for the Listing and Characterization of Impurities

[15] ICH Q1A Stability Testing of New Drug Substances and Products.

[16] Sharp, T.R. (Presentation) Forced Degradation: What? Why? How? FreeThink Technologies, Inc., Groton, Connecticut. http://www.cbinet.com/sites/default/files/files/Workshop\%20B(3).pdf

[17] Brown, R., Caphart, M., Faustino, P., Frankewich, R., Gibbs, J., Leutzinger, E., Lunn, G., Ng, L., Rajagopalan, R., Cjiu, Y. and Sheinin, E. (2001) Analytical Procedures and Method Validation: Highlights of the FDA's Draft Guidance.

[18] ICH Q1B, Photostability Testing of New Substances and Products.

[19] WHO (2005) WHO Guidance Document, Guideline on Submission of Documentation for Prequalification of Multi-Source (Generic) Finished Pharmaceutical Products (FPPs) Used in the Treatment of HIV/AIDS, Malaria and Tuberculosis-Guide Generic Submit Doc FPPs.

[20] Baertschi, S.W., Alsante, K.M. and Reed, R.A. (2011) Pharmaceutical Stress Testing Predicting Drug Degradation. Vol. 210.

[21] Alsante, K.M., Martin, L. and Baertschi, S.W. (2003) A Stress Testing Benchmarking Study. Pharmaceutical Technology.

[22] Pilaniya, K., Chandrawanshi, H.K., Pilaniya, U., Manchandani, P., Jain, P. and Singh, N. (2010) Recent Trends in the Impurity Profile of Pharmaceuticals. Journal of Advanced Pharmaceutical Technology \& Research, 1, 302-310.

[23] ICH Q2 Validation of Analytical Procedures.

[24] Fathima, N., Mamatha, T., Qureshi, H.K., Anitha, N. and Rao, J.V. (2011) 
Drug-Excipient Interaction and Its Importance in Dosage form Development. Journal of Applied Pharmaceutical Science, 1, 66-71.

[25] Bharate, S.S., Bharate, S.B. and Bajaj, A.N. (2010) Interactions and Incompatibilities of Pharmaceutical Excipients with Active Pharmaceutical Ingredients: A Comprehensive Review. Journal of Excipients and Food Chemicals, 1, 3-26.

[26] Janzen, H. (2016) Forced Degradation Studies-Comparison between ICH, EMA, FDA and WHO Guidelines and ANVISA's Resolution RDC 53/2015. Master of Drug Regulatory Affairs, Rheinische Friedrich-Wilhelms-University of Bonn, Bonn.

[27] European Medicines Agency (1994) Investigation of Chiral Active Substance. European Medicines Agency Guideline.

[28] FDA (1992) Development of New Stereoisomeric Drugs. FDA Guidances.

[29] Karnes, H.T. and Sarkar, M.A. (1987) Enantiomeric Resolution of Drug Compounds by Liquid Chromatography. Pharmaceutical Research, 4, 285-292. https://doi.org/10.1023/A:1016437018323

[30] Xiang, Y., Wu, N., Lippert, J.A. and Lee, M.L. (2002) Separation of Chiral Pharmaceuticals Using Ultrahigh Pressure Liquid Chromatography. Chromatographia, 55, 399-403. https://doi.org/10.1007/BF02492267

[31] Kamoto, Y. and Kaida, Y. (1994) Resolution by High-Performance Liquid Chromatography Using Polysaccharide Carbamates and Benzoates as Chiral Stationary Phases. Journal of the American Chemical Society, 666, 403-419.

[32] Subramanian, G. (1994) A Practical Approach to Chiral Separations by Liquid Chromatography. Vch Verlagsgesellschaft Mbh, Weinheim, Germany.

[33] Ahuja, S. (1997) Chiral Separations. ACS, Washington DC, Chapter 10, 271 p.

[34] Yashima, E. and Okamoto, Y. (1995) Chiral Discrimination on Polysaccharides Derivatives. Bulletin of the Chemical Society of Japan, 68, 3289-3307. https://doi.org/10.1246/bcsj.68.3289

[35] Yashima, E. (2001) Polysaccharide-Based Chiral Stationary Phases for High-Performance Liquid Chromatographic Enantioseparation. Journal of Chromatography A, 906, 105-125. https://doi.org/10.1016/S0021-9673(00)00501-X

[36] Tachibana, K. and Ohnishi, A. (2001) Reversed-Phase Liquid Chromatographic Separation of Enantiomers on Polysaccharide Type Chiral Stationary Phases. Journal of Chromatography $A, 906,127-154$. https://doi.org/10.1016/S0021-9673(00)00955-9

[37] Aboul-Enein, H.Y. (2001) High-Performance Liquid Chromatographic Enantioseparation of Drugs Containing Multiple Chiral Centers on Polysaccharide-Type Chiral Stationary Phases. Journal of Chromatography A, 906, 185-193. https://doi.org/10.1016/S0021-9673(00)00950-X

[38] Okamoto, Y., Kawashima, M., Yamamoto, K. and Hatada, K. (1984) Useful Chiral Packing Materials for High-Performance Liquid Chromatographic Resolution. Cellulose Triacetate and Tribenzoate Coated on Macroporous Silica Gel. Chemistry Letters, 13, 739-742. https://doi.org/10.1246/cl.1984.739

[39] Okamoto, Y., Kawashima, M. and Hatada, K. (1984) Chromatographic Resolution. 7. Useful Chiral Packing Materials for High-Performance Liquid Chromatographic Resolution of Enantiomers: Phenylcarbamates of Polysaccharides Coated on Silica Gel. Journal of the American Chemical Society, 106, 5357-5359. https://doi.org/10.1021/ja00330a057

[40] Okamoto, Y., Aburatani, R., Fukumoto, T. and Hatada, K.(1987) Useful Chiral Stationary Phases for HPLC. Amylose Tris(3,5-dimethylphenylcarbamate) and 
Tris(3,5-dichlorophenylcarbamate) Supported on Silica Gel. Chemistry Letters, 16, 1857-1860. https://doi.org/10.1246/cl.1987.1857

[41] Soma, I.V., Raghuram, R.P. and Sriramulu, J. (2011) Novel Chiral LC Methods for the Enantiomeric Separation of Bicalutamide and Thalidomide on Amylose Based Immobilized CSP. Current Pharmaceutical Analysis, 7, 47-53.

[42] Canada Health (2000) Guidance on Stereochemical Issues in Chiral Drug Development.

[43] USP <233> Elemental Impurities-Procedures.

[44] USP <232> Elemental Impurities-Limits.

[45] Raghuram, P., Soma Raju, I.V. and Sriramulu, J. (2010) Heavy Metals Testing in Active Pharmaceutical Ingredients: An Alternate Approach. Pharmazie, 65, 15-18.

[46] CDER (2016) Elemental Impurities in Drug Products Guidance for Industry. Draft Guidance.

[47] ICH (2014) Guideline for Elemental Impurities Q3D. ICH Harmonised Guideline.

[48] ICH Q3C Impurities: Residual Solvents.

[49] USP <467> Residual Solvents.

[50] Raghuram, P., Soma Raju, I.V. and Sriramulu, J. (2010) GC Quantification of Cyclopropylamine, Diethylamine and Triethylamine in Active Pharmaceutical Ingredients. Chromatographia, 71, 963-966.

[51] CDER (2009) Guidance for Industry Residual Solvents in Drug Products Marketed in the United States.

[52] USP <941> X-Ray Powder Diffraction.

[53] CDER (2007) Guideline on ANDAs: Pharmaceutical Solid Polymorphism Chemistry, Manufacturing, and Controls Information.

[54] CDER (2016) Draft Guidance on Regulatory Classification of Pharmaceutical Co-Crystals.

[55] Bauer, J.F. (2008) Polymorphism-A Critical Consideration in Pharmaceutical Development, Manufacturing and Stability. Journal of Validation Technology, 2, 15-23.

[56] ICH Q6A Specifications: Test Procedures and Acceptance Criteria for New Drug Substances and New Drug Products: Chemical Substances.

[57] Brittain, H.G. (2009) Polymorphism in Pharmaceutical Solids-Drugs and the Pharmaceutical Scienc. 2nd Edition, Vol. 192.

[58] PerkinElmer (2010) Raman Spectroscopy a Powerful Tool to Differentiate Pharmaceutical Polymorphs.

[59] (2009) Polymorphs of Fexofenadine Hydrochloride. United States Patent No. US2009/0149497A1.

[60] ICH M7 Assessment and Control of Dna Reactive (Mutagenic) Impurities in Pharmaceuticals to Limit Potential Carcinogenic Risk.

[61] ICH S9 Nonclinical Evaluation for Anticancer Pharmaceuticals.

[62] CDER (2008) Draft Guidance on Genotoxic and Carcinogenic Impurities in Drug Substances and Products: Recommended Approaches.

[63] ICH Q11 Guideline: Development and Manufacture of Drug Substances (Chemical Entities and Biotechnological/Biological Entities). Questions and Answers Version, 23 August 2017. 
[64] Benigni, R. and Bossa, C. (2006) Structural Alerts of Mutagens and Carcinogens. Current Computer-Aided Drug Design, 2, 169-176.

http://www.iss.it/binary/meca/cont/Ccadd2006\%20.1161263198.pdf

[65] EMEA (2007) Guideline on the Limits of Genotoxic Impurities. 